\title{
Jacques Cheyronnaud
}

\section{LA QUESTION DU TIMBRE}

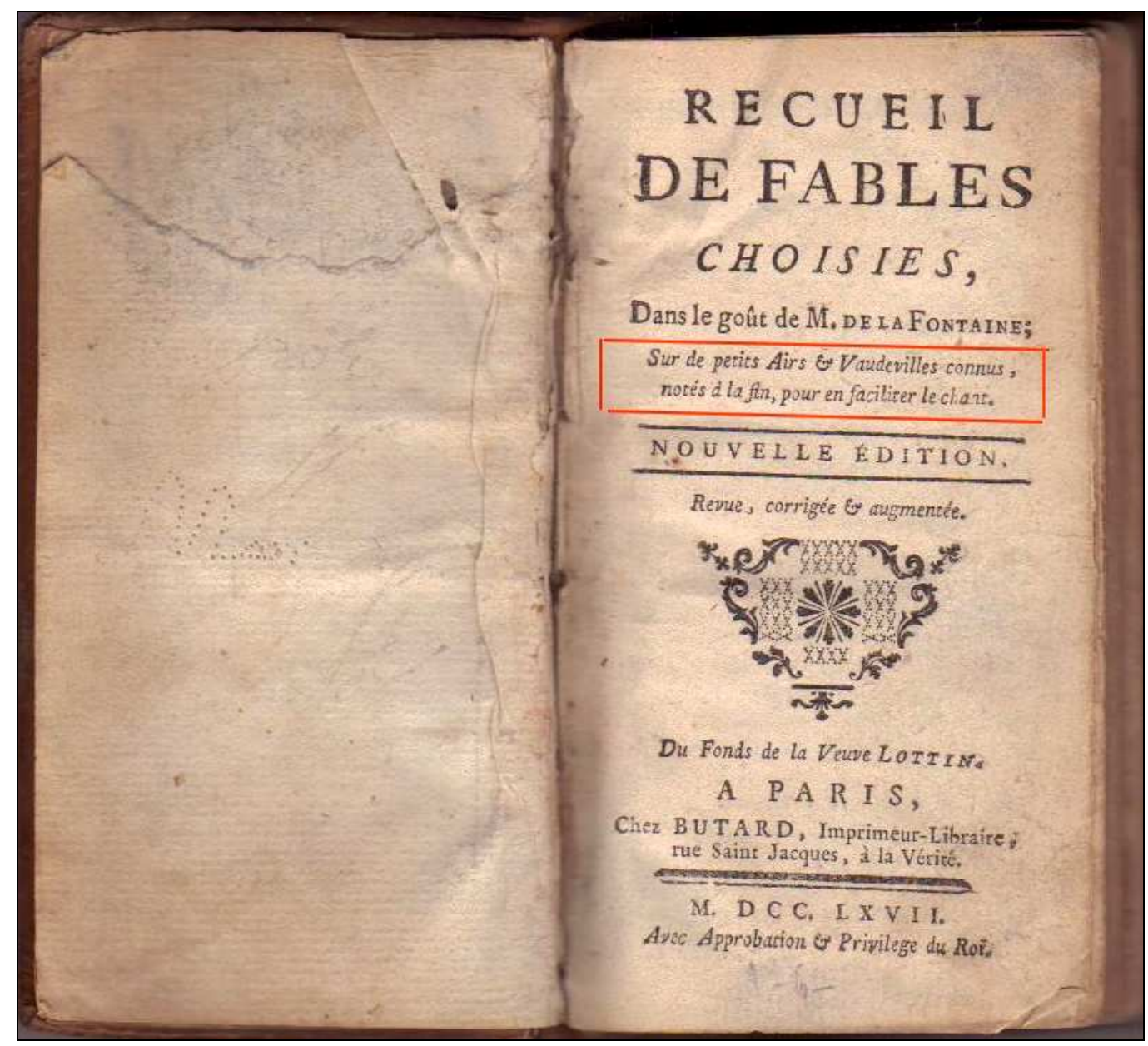

1. Recueil de Fables choisies dans le goût de M. de La Fontaine, par le Père Jean-Philippe Valette (1 ${ }^{\text {ère }}$ éd. : 1745)

Ces fables se chantent sur des timbres en vogue à l'époque

Conférence plénière

Mardi 28 octobre 2008

SOMMAIRE 


\section{Pour introduire}

1. Le format Chanson

2. L'individuation mélodique

3. D'une pratique, longtemps ordinaire

\section{Qu'est-ce qu'un timbre?}

1. La mention : «Air de »

2. Plusieurs formules désignatives pour un même air

\section{Des airs de parodie}

1. Question d'étymologie

2. «Saisir le goût et le caractère » de l'air

3. Associations secondes

\section{Le XIXe siècle chansonnier. Déplacements et transformations}

1. Scénographies de poche

2. L'entrepreneur et l'artisan

\section{5. «Territoires hymnodiques »}

1. «Tous-ensemble ». D'une solution profératoire collective

2. S'équiper de répertoires

\section{Pour conclure. La guerre des timbres}

1. Scénarités vocales

2. Parodies polémiques

\section{NOTES}

\section{Pour introduire}

Le paysage actuel de la production chansonnière en France est dominé par ce que l'on appellera le format chanson, à individuation mélodique. Qu'entend-on signifier par là ?

\section{Le format chanson}

Nous rangeons facilement sous l'appellation «chanson » des objets littéraires variés qui dans le projet de leurs auteurs, ne recourent pas toujours directement ou impérativement, aux ressources d'une mélodie. «Chansons », dit-on alors, que celles des Brel, Brassens et de tant d'autres... Mais aussi : Chansons des rues et des bois (Victor Hugo), Chanson du mal aimé (Guillaume Apollinaire), La bonne chanson (Verlaine)... Dans notre manière commune ou familière de parler de la chanson, nous donnons volontiers priorité à un objet textuel, un certain type de texte voire de disposition formelle littéraire, la dimension musicale et chantée venant souvent au second plan et facilement considérée comme accessoire.

Mais si «chanter une chanson» est bien articuler, découper ce texte, ce ne sera plus de la même manière que de le lire, ou même que de s'en saisir (également vocalement) pour le réciter ou le déclamer. Dans la présente intervention, on ne saurait se donner pour thématique la question de la chanson et de la pratique du timbre sans devoir problématiser, fût-ce schématiquement, ce déploiement ou cet acheminement en mode 
chanté de l'objet textuel comme clause intégrée au fonctionnement même de l'objet textuel tel qu'il a pu être anticipé, réfléchi par son auteur. Il nous faut donc inscrire ce fait qu'un auteur puisse programmer un certain type de «rendement» de ce qu'il signe, en premier lieu par un acheminement vocal verbal en régime de chant. En somme, il s'agit d'intégrer dans notre analyse le paramètre d'une énonciation chansonnière, c'est-à-dire : le procès même, effectif et concret, situé, de prise en charge vocale corporelle du texte par un «sujet chantant». Bref, ce qui fait qu'une « chanson » est bien ... une chanson.

Suggérons ceci. Le «chant de la chanson», si l'on ose dire, sera posé comme un moment de réalisation vocale effective de l'objet textuel, qui vient le déplacer de son statut assertif pour le mettre en position «d'énoncé rapporté », à la manière d'une citation ou de guillemets. Il est, si l'on veut, une certaine manière de dire les mots : manière de jouer vocalement sur leur surface, de les mettre en scène jusqu'à les étirer, à leur donner de l'élasticité. Pour la célèbre « diseuse fin de siècle», Yvette Guilbert (1867-1944), chanter devait être cet Art de dire,

Art d'allumer et d'éteindre les mots - de les plonger dans l'ombre ou la lumière selon leur sens, de les amoindrir ou de les amplifier - de les caresser ou de mordre de les sortir ou de les rentrer - de les envelopper ou de les dénuder, de les allonger ou des réduire (Y. Guilbert, L'art de chanter une chanson, 1928, pp. 11-12).

Sans renier pour autant l'emploi du terme même de «chanson » mais pour intégrer cette clause d'une ressource mélodique dans l'acheminent vocal et le déploiement perceptif sonore d'un texte comme partie intégrante des conditions de son rendement (celles programmées par un auteur), on emploiera spécifiquement l'expression : format chanson ou format. Ainsi désignera-t-on par là toute chanson en son immanence propre d'organisation métrico-rythmique, phonique, strophique ou non (la strophe y prend nom de couplet), avec ou sans refrain, et qui emprunte expressément aux ressources d'une cinétique musicale (la direction qu'imprime une dynamique de vections mélodiques et rythmiques), qu'il s'agisse d'une mélodie originale ou d'un timbre. Prenons, par exemple, la chanson Les feuilles mortes (paroles de Jacques Prévert, musique de Joseph Kosma). On peut en déclamer le texte que l'on appellera «poème » ou «chanson ». Selon la distinction méthodologique que l'on propose ici : lorsque l'on considèrera cet objet mélodico-textuel en tant que tel, c'est-àdire comme un texte équipé d'une mélodie pour une prise en charge vocale chantée ; on parlera ainsi du format Les feuilles mortes.

\section{L’individuation mélodique}

Autre proposition méthodologique pour cette intervention. Les formats chanson aujourd'hui les plus fréquents dans le paysage de la production chansonnière, sont marqués par le trait d'une individuation mélodique. A la différence d'une chanson sur timbre, entendons par là ce procédé qui consiste à équiper un texte d'une mélodie spécifiquement composée pour lui, soit par l'auteur des paroles, soit en collaboration avec un compositeur. Le format s'offre dès lors avec une mélodie en propre (on dit généralement «mélodie originale »), le tout pouvant être référencé par un intitulé comme Les feuilles mortes, un nom d'auteur (Jacques Prévert), un nom de compositeur (Joseph Kosma), voire une date de création (sa «première » en audition publique). Ce même format chanson, ainsi doté de sa mélodie originale, pourra se prêter à de nouvelles paroles, sérieuses ou plaisantes (le plus souvent, on dit alors «sur l'air de », ou «sur la chanson» des Feuilles mortes). Ainsi parlerat-on du timbre Les (ou Des) feuilles mortes. 


\section{D'une pratique, longtemps ordinaire}

Ce procédé désormais communément répandu et dominant qui consiste à doter un texte, une poésie, d'une mélodie originale et à garantir à ce type de format une protection juridique (ce que l'on appelle le droit d'auteur), semble marginaliser ou minorer cet autre, cependant historiquement prégnant dans notre patrimoine chansonnier : équiper un texte, une poésie d'un air déjà existant. Loin de constituer quelque anomalie morale qu'aujourd'hui on appellerait juridiquement «plagiat » ou «faux », ou de se cantonner aux trouvailles ludiques, parodiques, fort prisées de collectifs manifestant sur la voie publique, la chanson sur timbre a constitué une technique longtemps ordinaire, largement mise en œuvre et diffusée dans l'espace chansonnier au cours des siècles précédents.

Il faudra attendre la seconde moitié du XIXème siècle pour que le format à individuation mélodique devienne prédominant, dans le paysage de la chanson. Le développement d'un marché des spectacles de caféconcert le fera s'émanciper (on parle à l'époque de chansonnette) jusqu'à constituer un enjeu économique, en même temps qu'il va profondément modifier le paysage des pratiques chansonnières. Tandis que cette tendance rédactionnelle et compositionnelle s'émancipera jusqu'à se systématiser, la pratique du timbre jusqu'ici communément partagée, cultivée, se déplacera en se spécialisant ou se «sectorisant ». Elle se maintiendra sous l'image d'un «artisanat culturel» (volontiers anti café-concert dans la seconde moitié du XIXème siècle), aux mains de collectifs, par exemple d'associations d'amateurs se recommandant des riches heures du Caveau ou de la Lice chansonnière (1). Cette pratique du timbre se retrouve également ou se continue dans la même seconde moitié du XIXème siècle dans un volontarisme chansonnier campé au cœur des épreuves collectives, sociales et politiques de la société (2). Ou encore au cabaret (où se maintient toujours le terme de «chansonnier »), sous la forme de parodies satiriques ou burlesques. Elle subsiste de nos jours, souvent comme pratique privée, plaisante et conviviale comme à l'occasion de repas de mariages ou de fêtes, ou comme forme ludique de la critique dans l'entre-soi de groupes restreints ou fermés, ou dans des pratiques collectives de protestation lors de manifestations de rue.

La présente intervention sera consacrée tout d'abord à une présentation de la pratique du timbre dans l'espace chansonnier des XVIIIème et XIXème siècles. On s'attachera également à cerner quelques unes des transformations de cet espace dans la seconde moitié du XIXème, qui vont faire de la chanson sur timbre non tant une pratique déclassée que dirigée, valorisée et cultivée aux marges d'un paysage dominé par le marché de la production de formats à individuation mélodique. On s'arrêtera brièvement sur l'un des secteurs pour lequel cette pratique s'exercera à dessein : celui des «hymnodies » ou mieux des «territoires hymnodiques » religieux, politiques ou partisans (partis politiques), scolaires, etc.

\section{Qu'est-ce qu'un timbre?}

\section{La mention : «Air de »}

Sur la page imprimée d'un format chanson qui emprunte aux ressources d'un air préexistant, comment s'y prenaient nos chansonniers pour permettre au lecteur d'identifier l'air ou les airs, car ils pouvaient en proposer plusieurs à la fois, qu'ils avaient choisis pour leur texte, sans que ce lecteur ait nécessairement à passer 
par la mélodie notée ? La mention de l'air choisi était portée sous le titre de la chanson; cette mention comprenait une courte locution du type Air : ou Air de, ou Sur l'air de, suivie d'une formule désignative.

Ainsi le cas du timbre Ah! Daignez m'épargner le reste sur lequel se chante, dans l'exemple ci-dessous, la chanson Les deux cousins de Pierre-Jean de Béranger :

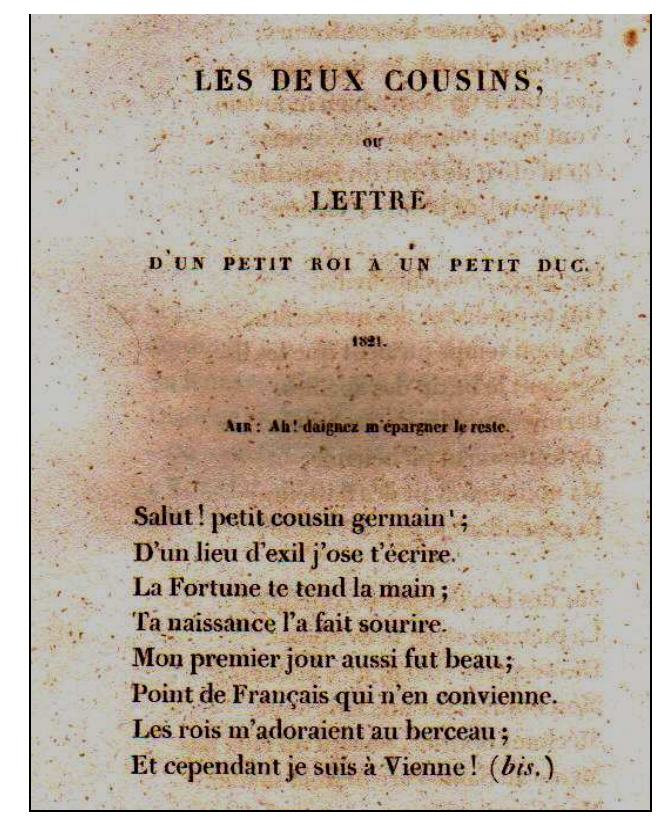

2. Extrait de la chanson Les deux cousins (1821) de Pierre-Jean de Béranger (1780-1857) Mention du timbre Ah? Daignez, m'épargner le reste

La mention de l'air sélectionné par Béranger, l'auteur des paroles, est portée sous l'intitulé de la chanson; elle comprend le syntagme Air : suivi de la formule désignative : Ah! Daignez m'épargner le reste.

D'où provient la formule? D'un air très en vogue à l'époque, extrait des Visitandines, comédie ou opéra comique en deux actes, de Louis-Benoît Picard (1769-1828) pour le livret et de François Devienne (1759-1803) pour la musique (composition originale). L'air se présente comme suit (version de la Clé du Caveau, air $\left.n^{\circ} 12\right)$ :

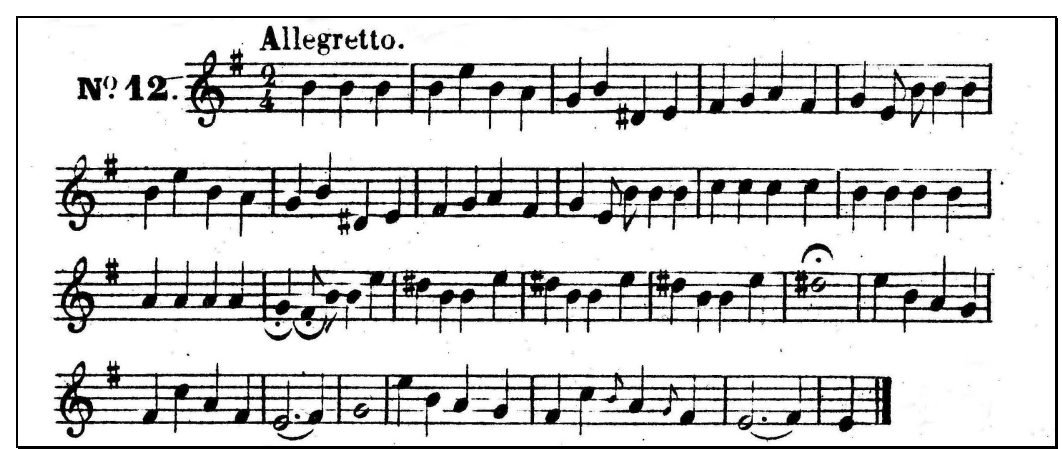

3. Air de la fin de la sc.3, Acte II des Visitandines (Clé du Caveau, air n 12)

Cet air des Visitandines sert à deux couplets :

Ah ! De quels souvenirs affreux

Votre demande m'a frappée ;

Un jour nous nous connaîtrons mieux :

Vous saurez comme on m'a trompée.

Le ciel en nous donnant un cœur,

Nous fît un présent bien funeste.

Vous m'entendez, ma chère sœur, 


\section{Daignez m'épargner le reste. (bis) \\ Dans cette maison à quinze ans, Je n'étais que pensionnaire ; Un jeune abbé des plus charmants Logeait au prochain séminaire ; Un certain jour il vint me voir, Il avait un air tout céleste, \\ Et sans la grille du parloir, \\ Daignez m'épargner le reste. (bis)}

On le voit, la formule désignative évoquée jusqu'à présent pour porter l'air en question, reprend le dernier vers bissé de chaque couplet; vers qui fait office de refrain. Mais à l'époque, dans diverses occurrences de réutilisation de cet air, deux autres vers de ces deux mêmes couplets peuvent également servir de formules désignatives : le premier vers de chacun des deux, soit : Ah! De quels souvenirs affreux et Dans cette maison à quinze ans.

\section{Plusieurs formules désignatives pour un même air}

L'exemple précédent pointe une difficulté plus générale en la matière, celle de possibles occurrences d'un seul et même air sous des formules désignatives différentes. Ou, si l'on veut: difficulté d'une identification d'origine d'un air à partir d'une association qui n'est pas nécessairement stable ou durable à telle ou telle formule désignative. Dans l'exemple précédent, les trois formules désignatives sont portées par le seul et même texte de deux couplets, dont elles reprennent chacune un vers différent. On notera que si Béranger emploie bien la formule désignative Daignez m'épargner le reste dans l'exemple proposé précédemment Les deux cousins (ainsi que pour sa chanson Le contrat de mariage), il mentionne la formule Dans cette maison à quinze ans comme premier timbre à la chanson Les cinq étages - qui utilise cependant le même air que les deux précédentes :

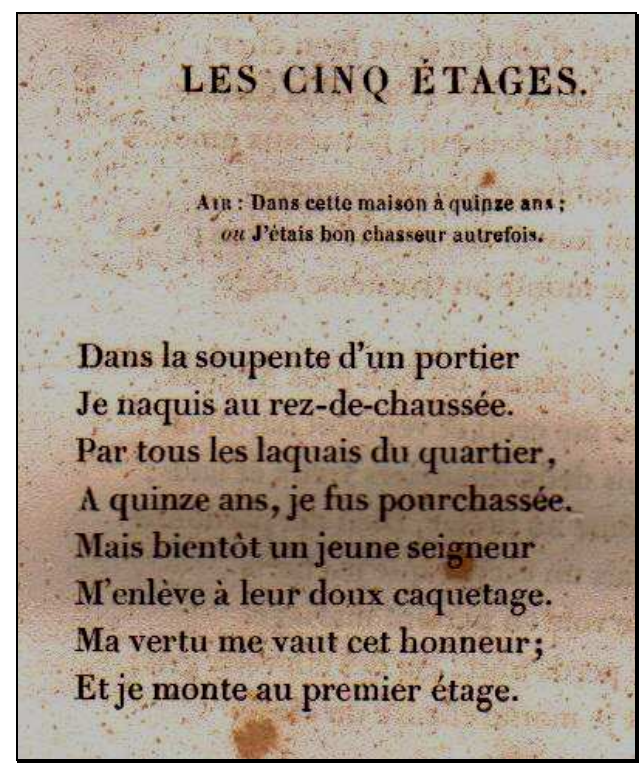

4. Extrait de la chanson Les cinq étages de P.-J. de Béranger.

Mention du même air (cf. cliché 3), avec comme première formule désignative : Dans cette maison à quinze ans 
Observons également, d'une part, que la pièce Les Visitandines offrira plusieurs autres timbres ; d'autre part, que leurs formules désignatives ne sont pas nécessairement ou pas seulement le premier vers d'un premier couplet. Ainsi par exemple, rencontrera-t-on pour un même air, tel dans la scène 5 de l'Acte I, des mentions telles que «Air : Enfant chéri des dames» (formule d'incipit) ou « Air du Rondeau des Visitandines» ou « Air : Rondeau de Belfort ». De même, pour un autre air de la même scène, les mentions «Air : Dans l'asile de l'innocence » (formule d'incipit) ou «Air de la Romance d'Euphémie ». Soit encore, pour d'autres timbres de la même pièce, les mentions «Air: A moins que dans ce monastère» (formule d'incipit) ou «Air du vaudeville des Visitandines », les deux référant au final de la pièce. Tous ces airs sont répertoriés dans la Clé du Caveau.

Sur ce point, toute systématisation s'avère difficile. On dira en résumé que dans une mention de timbre, la formule désignative peut porter les premiers mots ou l'un des vers d'un couplet (souvent le premier vers ou incipit littéraire) ou du refrain, ou un titre de chanson, ou l'un des genres à l'intérieur d'une même pièce tels rondeau, romance, vaudeville (3), etc. Les cas sont multiples et complexes, tant un même air a pu être porté successivement ou parfois dans le même temps sous diverses formules désignatives - ou certaines mêmes formules désignatives, porter des airs différents. Ainsi de l'illustre air connu sous la formule désignative Au clair de la lune dont la fixation définitive sous cette formule se stabilisera au XIXème siècle à force de citations et de réimpressions. On pouvait auparavant le rencontrer sous d'autres formules comme (En) Roulant ma brouette (dernier quart du XVIIIème siècle) (4).

Ajoutons ceci. Dans la logique de ce système général de l'emprunt mélodique - c'est-à-dire, cette pratique commune du timbre, donc de circulation des airs -, quoi de plus évident que ce principe de substitution de nouvelles paroles puisse éventuellement s'étendre aux formules désignatives elles-mêmes ? N'était-ce pas là d'ailleurs, une visée morale de la part de nombreux pédagogues, qu'ils soient de religion ou de la Révolution, que d'épurer le secteur de la chanson et des «paroles frivoles », en leur offrant des remplaçants (5) : «tuer le vice avec ses propres armes »?

\section{Des airs de parodie}

\section{Question d'étymologie}

Si le terme de timbre au sens d'air préexistant adapté à de nouvelles paroles se rencontre au moins à la fin du dix-huitième siècle comme chez le chansonnier vaudevilliste Pierre Laujon (6), il n'en renvoie pas moins à une forme ancienne, bien identifiée notamment pour ce qui concerne le domaine musical : la parodie (ou contrafacture).

Mais regardons l'étymologie de «parodie», et ne versons pas trop vite ce terme dans le champ sémantique de la plaisanterie ou de la dérision comme nous le faisons de nos jours. Si le terme grec odos désigne l'élément formel (chant, texte), celui de para peut avoir deux sens tels que : «à côté de » ou «le long de » (comme dans paraphrase) et «à l'encontre de» (comme dans paradoxe). Il nous faut compter avec cette première dimension, en quelque sorte neutre, du terme qui renvoie à un principe d'appropriation d'un antécédent par adaptation ou substitution - ici, adaptation ou substitution d'un nouveau texte à un ancien, tout en lui conservant le même support mélodique. Cette dimension neutre sera conservée dans le secteur chansonnier, parallèlement au sens d'une pièce à charge ou à vocation plaisante ou satirique. Ainsi de cette dimension neutre, comme chez Jean-Jacques Rousseau dans son Dictionnaire de musique (1767) : 
«Parodie : Air de symphonie dont on fait un air chantant en y ajoutant des paroles. Dans une musique bien faite le chant est fait sur les paroles, et dans la parodie les paroles sont faites sur le chant: tous les couplets d'une chanson, excepté le premier, sont des espèces de parodies. »

De même pour P. Laujon, auteur déjà évoqué : on devrait distinguer (en théorie du moins) la «chanson proprement dite », pour laquelle les vers sont mis en musique et «soumis aux règles ordinaires de la poésie » et la « Parodie ou Chanson parodiée » pour laquelle

«Le poète a le double mérite d'avoir choisi l'air nécessaire pour bien exprimer son idée, d'en avoir indiqué le caractère, de s'être asservi fidèlement à la prosodie musicale, d'avoir le premier fixé les césures, les hémistiches de chaque vers, etc. : aussi /.../ quand ce dernier genre est celui des deux que nous pratiquons de préférence, je dois vous faire observer que s'il admet plus de difficultés, il peut aussi se permettre des licences qui lui sont particulièrement affectées ; guidé par la mesure de l'air, depuis le monosyllabe jusqu'à celle qui termine le vers alexandrin, le parodiste peut employer pour vers une, deux, trois syllabes, etc., se passer de césures, n'admettre que celles indiquées par l'air: ces licences, que réprouve la poésie, n'ont rien qui blesse l'oreille, ni qui soit défectueux dans la parodie ; elles sont même nécessaires dans presque toutes les parodies des grands morceaux de musique, opéras, ouvertures, menuets, etc.»(P. Laujon, Euvres choisies, 1811, tome IV, pp. 108-109).

Entre «chanson proprement dite » et «parodie ou chanson parodiée », il y a donc cette différence qui, pour la seconde, impose à un auteur qu'il s'ajuste aux contraintes formelles portées par le modèle de timbre dont il compte tirer parti. La première relèverait du régime de l'individuation mélodique (ce qu'on appellera parfois « ariette » dans la seconde moitié du XVIIIe siècle).

\section{2. « Saisir le goût et le caractère » de l'air}

Trois verbes pourraient résumer l'exercice chansonnier de l'adaptation à un précédent que constitue la chanson sur timbre ou parodie (P. Laujon): «ajuster », « adopter », « associer ».

Ajuster. Il s'agit d'abord de se régler au mieux sur un modèle de format chanson qui porte avec lui en premier lieu une certaine coupe, c'est-à-dire une organisation formelle : structure strophique (comprenant ou non un refrain articulé avec la strophe ou une simple reprise de vers dans la strophe même, jouant ainsi le rôle de refrain), nombre de vers dans la strophe, disposition des césures, nombre de pieds ou syllabes dans le vers, jeu de terminaisons assonantiques (rimes en fin de vers et jeux des rimes entre elles). Compter juste (comptage syllabique), quitte à user de recettes tel le «remplissage syllabique » avec des «Oh!» « Ah!», « Hélas!», «Amis », etc., et «courir après la rime» constituent des opérations qui pourront faire de l'ajustement un divertissement collectif.

Adopter. Mais l'exercice chansonnier de la chanson sur timbre ne se limitera pas seulement à ces opérations d'ajustement technique formel à un modèle préexistant. Par exemple, le souci des auteurs de «chansons spirituelles» ou cantiques (le genre est un haut lieu de cette parodie «neutre» au XVIIIe siècle) d'assortir l'air aux nouvelles paroles :

Nous connaissons tous la difficulté de l'entreprise ; pour peu qu'on ait quelque teinture de la poésie \& de la musique, on doit concevoir combien il est difficile d'appliquer, avec succès, des paroles à des airs déjà faits. Le choix d'un sujet analogue à celui du chant ; la nécessité presque continuelle où l'on est d'employer des syllabes longues ou brèves, qui répondent à la différente valeur des notes, la brièveté de la plupart des vers, qui amène trop tôt la rime, sans vous laisser le 
temps de la préparer, ni la liberté d'étendre une pensée, ou de suivre un sentiment, l'attention scrupuleuse qu'on doit avoir à saisir le goût \& le caractère d'un récit, d'une ariette, d'un rondeau, et d'en rendre le mouvement, la cadence, les agréments ; tout concourt à gêner le travail de poète \& le force à, sacrifier bien des vers qui pourraient être harmonieux à la lecture et à la déclamation, mais qui seraient rudes à l'oreille, \& peu propres à être chantés. (Opuscules sacrés et lyriques ou Cantiques /.../ à l'usage des Catéchismes de la Paroisse de Saint Sulpice, 1772, p. XIV).

Ici, la question est celle du choix de l'air d'un timbre : faire en sorte que les nouvelles à portée morale puissent tirer profit d'un «éthos » de l'air, de sa manière d'être ou de son allure, et qu'aucun, de l'air et des nouvelles paroles, ne vienne grever l'économie formelle ou de contenu de l'autre.

D'autres, moins préoccupés par le contenu grave ou sérieux comme dans le cas des vaudevilles, n'insistent pas moins sur l'une des conditions de réussite de cette adoption de l'esprit d'un air que l'on emprunte : maintenir ce caractère approprié du nouveau à l'ancien en lui sauvegardant son économie formelle, un « dispos » qui donne à la parodie cette « rondeur » d'un « genre agréable » que l'on aime chanter et que l'on peut retenir. Ainsi André-Ernest-Modeste Grétry :

Qu'est-ce que la tragédie? Qu'est-ce que le genre agréable ? On aurait vu, si quelqu'un avait su le dire, que la déclamation de ces deux genres de drames n'est pas la même, non plus que la musique; que la tragédie ne dit, le plus souvent, qu'une fois les choses, mais avec énergie et brièveté ; que le genre agréable, au contraire, prend les détours des grâces, de l'esprit, du sentiment; qu'il répète trois fois les mêmes idées sous des formes différentes, et par conséquent use souvent des retours périodiques. Je dis donc que la rondeur, soit dans le discours, comme dans tous les arts, est la forme d'adoption ; Tout ce qui est beau, est rond ou à-peuprès, au physique comme au figuré /.../ rond; carré ou symétrique sont des synonymes dans les arts /.../ La rondeur, des retours de phrase en musique, en font presque tout le charme /.../ Qu'est-ce qui fait le charme de nos vaudevilles que l'on chante depuis un siècle ? C'est leurs retours, leurs répétitions, leur rondeur /.../ Gluck aimait les bons vaudevilles, c'est-à-dire ceux qui, par leur parfaite construction, se logent dans la mémoire des hommes pour plusieurs siècles (Grétry, Essais sur la musique, 1797, 2, pp. 73-76).

\section{Associations secondes}

Enfin, associer. Une autre part de réussite (ou de «rendement») d'une parodie pourra résider dans l'effet produit par le rapprochement entre nouvelles et anciennes paroles, et la pratique ou l'expérience que le lecteur ou le public saura effectuer de lui-même grâce à ses connaissances ou ses souvenirs. Pourquoi un auteur aura-t-il préféré tel timbre à tel autre, de même coupe ou de coupe différente ? Pourquoi Béranger a-t-il préféré, pour sa chanson Les cinq étages évoquée précédemment, la formule désignative Dans cette maison à quinze ans à cette autre Daignez m'épargner le reste, qu'il connaissait fort bien pour l'utiliser également avec le même air en d'autres chansons?

Au delà des avantages formels de la coupe que peut porter avec lui un timbre et de l'imitation que peut en faire l'auteur jusque dans un alignement serré : même refrain, mêmes mots-clés, mêmes jeux de rimes, etc., il faudrait ici prendre en compte cette possibilité d'un choix prémédité de l'auteur de nouvelles paroles cherchant à tirer parti, pour le rendement de sa chanson, de ce que le modèle ainsi emprunté peut représenter ou évoquer auprès du lecteur ou du public. Le rapprochement ou le contraste créés peuvent ainsi constituer une part - implicite ou non dite - du sens de la chanson : cette part d'achèvement que confie

l'auteur à son lecteur ou son public (comme dans toute parodie) et qui relèvera des compétences de chacun à 
savoir reconnaitre, effectuer le rapprochement. On retrouverait ainsi le terme de parodie en tant que charge plaisante ou satirique certes, mais en premier lieu en tant qu'écriture connotative : le jeu sur des associations secondes (7). Et si nombre de textes que vient servir un timbre constituent de ces productions qui visent expressément, avec le concours de la formule désignative, et dans un contexte précis, à «mettre au rire » («ridiculiser ») tel comportement, telle figure ou tel personnage, telle fonction ou tel événement, etc., d'autres productions sur timbre visent au contraire le sérieux ou la gravité. Certaines parodies de répertoires cultuels (culte catholique, par exemple) de la période de la Révolution française ne visent pas nécessairement la dérision : elles entendent surtout profiter de l'autorité ou de la prégnance de répertoires, pour souligner l'importance ou mieux diffuser les contenus qu'elles véhiculent (8).

Et méfions-nous en ce domaine musicologique. Nous aurions sans doute beaucoup plus tendance de nos jours à trouver incongru et à sourire que dans tel recueil de cantiques ou «chansons spirituelles » du XVIIIème siècle, un cantique catholique pour le lundi de Pentecôte porte la mention du timbre J'avais promis à ma maitresse, ou tel autre pour une retraite de première communion, le timbre Prends ma Philis, prends ton verre. Nombre d'éditeurs, d'auteurs ecclésiastiques ou autres, on l'a évoqué, se plaisent volontiers à pratiquer l'exercice, comme l'abbé Simon Joseph Pellegrin (1663-1745).

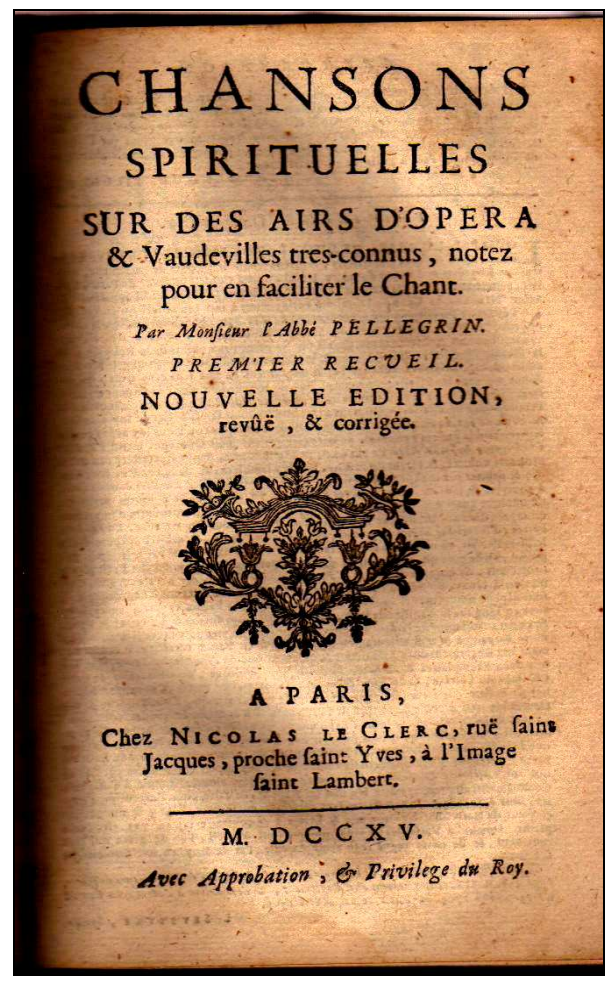

5. Les chansons spirituelles ou cantiques, haut lieu du timbre au XVIIIème siècle.

L'un des nombreux recueils de l'Abbé Pellegrin (1715)

Mais au XVIIIème siècle, cet objet de religion n'a pas le statut cultuel qu'il gagnera plus tard : à l'époque, «chansons spirituelles» ou cantiques sont partie intégrante de l'espace chansonnier et de la pratique domestique; ils relèvent de sa logique générale de l'emprunt à travers le timbre. Le cantique sur timbre sera encore largement pratiqué chez nous dans la première moitié du XIXème siècle, des compilateurs ou des éditeurs n'hésitant pas à renvoyer à la Clé du Caveau. Ainsi, parmi bien d'autres recueils, celui intitulé Nouveau choix de 
cantiques de Saint-Sulpice à l'usage des catéchismes de cette paroisse parisienne, se diffusera abondamment dans l'ensemble des diocèses de France et sera régulièrement réédité durant la première moitié du même siècle.

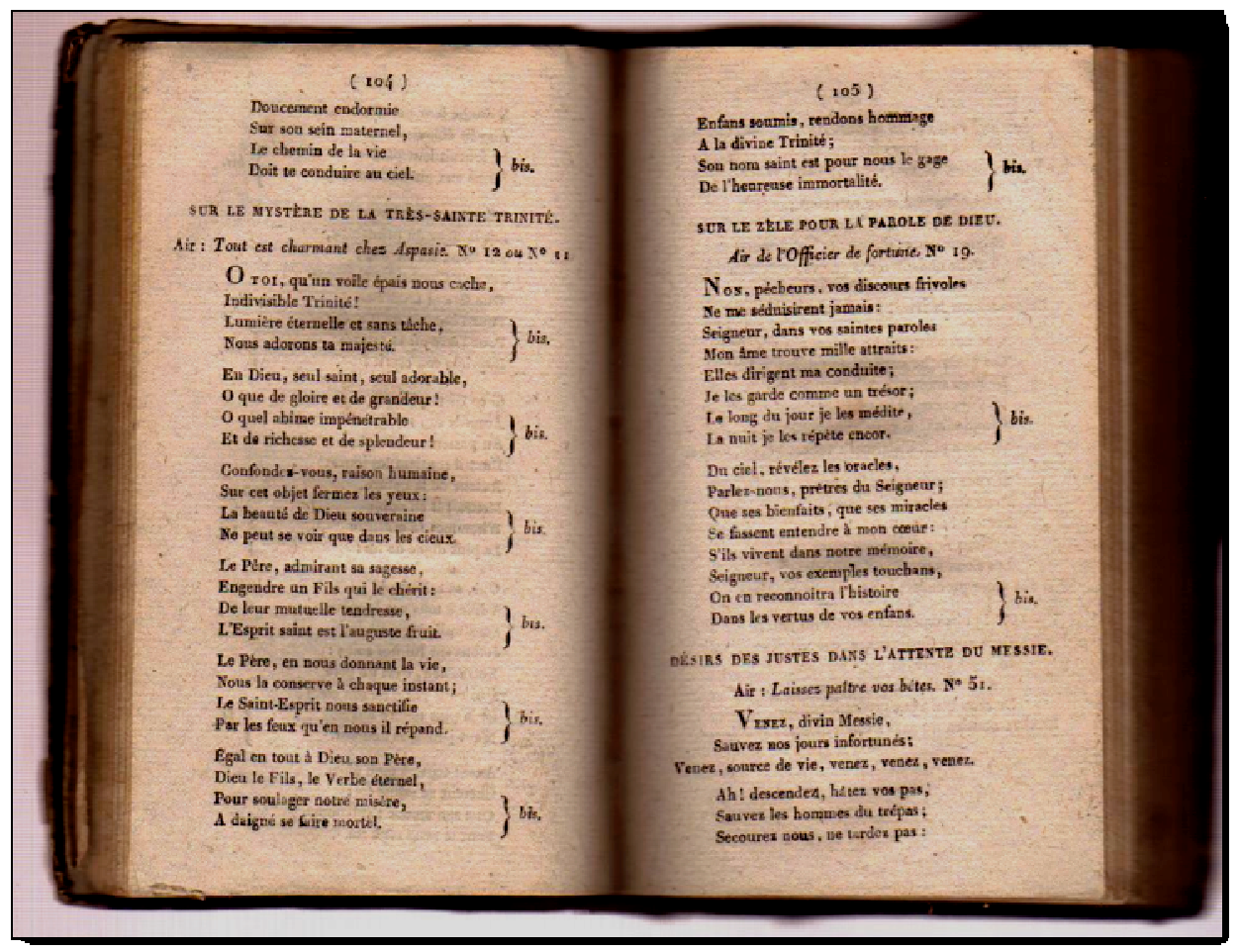

6. Nouveau choix de cantiques de Saint-Sulpice (1817). Un recueil qui sera abondamment réédité dans la première moitié du XIXème siècle et largement diffusé en France.

\section{Le XIXe siècle chansonnier Déplacements et transformations}

On l'a évoqué en introduction, le paysage de la production chansonnière au XIXe siècle va connaître d'importants aménagements liés au développement, d'une part des arts et des lieux de spectacle (ici, les cafésconcerts) sous le second Empire et plus encore sous la Troisième République, et d'autre part, à partir de la Restauration, de l'installation durable dans le paysage de la critique politique, d'un «volontarisme chansonnier» pour lequel les grandes crises politiques et sociales, en premier lieu les événements de 1830, 1848 et 1870-71, constitueront des « espaces » d'émulation.

\section{Scénographies de poche}

Deux dates peuvent nous servir de repères : 1851 et 1867.

Du jour où le parolier et dramaturge Ernest Bourget (1814-1864) qui réclamait depuis des années le respect du principe du droit d'auteur dans les cafés-concerts, eut gain de cause devant les tribunaux et décida, le 18 mars 1850, de fonder avec quelques autres un Syndicat des Auteurs, Compositeurs et Editeurs de musique qui devint un an plus tard, en 1851, une Société du même nom : la SACEM, la chansonnette allait avoir de beaux jours devant elle. Jusqu'ici en concurrence directe avec la pratique du timbre dans la culture de la «chanson vaudeville » du siècle précédent, le format à individuation mélodique passerait définitivement au devant de la 
scène chansonnière. Non seulement le produit de cette collaboration serait désormais protégé mais la multiplication des établissements ouvrait un important marché des spectacles de café-concert.

D'autant que quelques années plus tard, le 31 mars 1867, le directeur de l'administration des théâtres, Camille Doucet, donnerait enfin à ces établissements l'autorisation de présenter des artistes «travestis» (en costumes de scène), de monter de petites pièces, des pantomimes, des numéros de danse, de changer les décors... Le Second Empire, plus encore la Troisième République vont constituer d'importants moments d'expansion d'un modèle « café-concert (9) ».

Les conditions seront désormais réunies pour l'émancipation de ce type de format, la chansonnette, en scénographie de poche, portée par une figure qui pour émerger face à une concurrence de plus en plus forte devra mettre au point une individualité stylistique (silhouette, jeu de scène, manières de voix et de chant, etc.), d'autant recopiée, imitée par les débutants. Un troisième personnage va s'imposer à part entière aux côtés de l'auteur et du compositeur : l'interprète, à l'épreuve de la scène, d'un auditoire et de salles de mieux en mieux aménagées et réservées à cet effet.

La chanteuse-interprète Thérésa (Emma Valadon, 1837-1913), au zénith de sa renommée dans la décennie soixante, fait ici figure d'ancêtre - la première, considère-t-on souvent - d'un profil de vedette en la matière. Autre exemple, parmi bien d'autres, de la mise au point de cette individualité stylistique au caféconcert, le chanteur interprète Félix Mayol (1872-1841), qui soignera sa silhouette (habit, brin de muguet à la pochette, toupet), connu pour ses mimiques et son jeu de mains - ce qui lui vaudra d'être l'interprète attitré de la célèbre chanson Les mains de femmes (paroles : Emile Herbel, musique : Désiré Berniaux, 1907) spécifiquement composée pour lui.

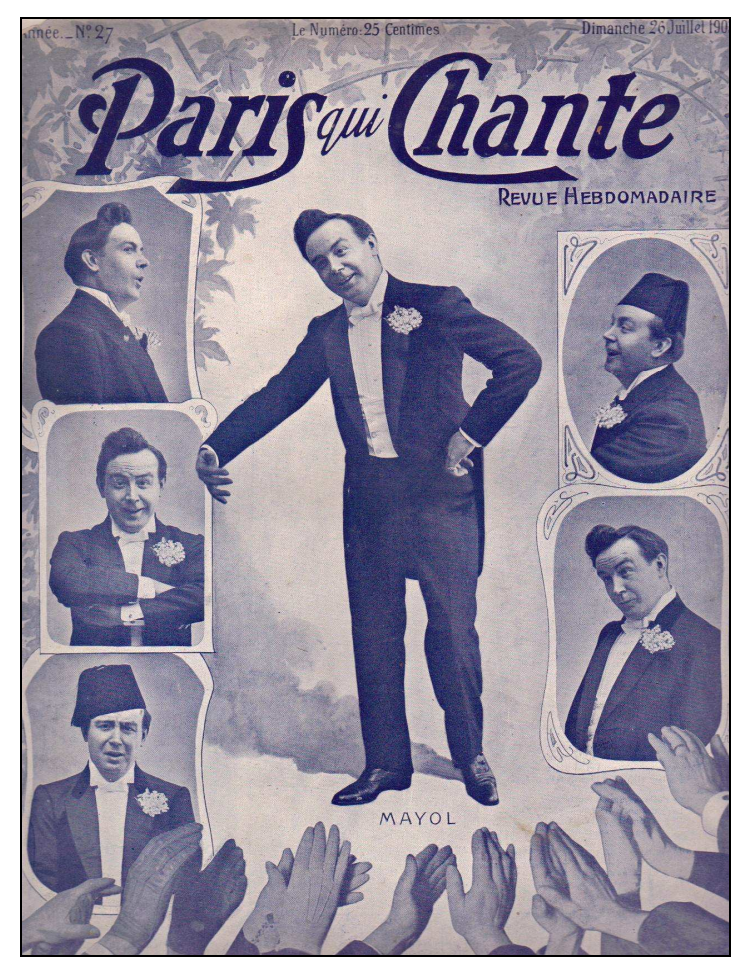

7. Individualités stylistiques. Le chanteur interprète Mayol (1903) : costume, toupet, brin de muguet, gestique, etc. figurent parmi les traits distinctifs progressivement mis au point à des fins de carrière. 


\section{L'entrepreneur et l'artisan}

Ainsi se configure, dans cette seconde moitié du XIXème siècle, un marché du café-concert - de la chansonnette et des droits d'auteur -, en même temps qu'un espace de «professionnalisation » regroupant un ensemble d'activités auxquelles on peut consacrer une certaine durée qui permette d'en retirer quelque revenu, jusqu'à pouvoir en vivre si l'on sait y faire. Un espace qui sera d'autant lucratif pour peu qu'on regroupe ces activités entre elles, qu'on les monopolise en en maîtrisant la chaîne de la confection à la diffusion en passant par l'édition graphique, la répétition ou l'apprentissage à des interprètes, jusqu'à l'organisation de tournées de troupes ou d'artistes.

Deux tendances, quasiment deux esthétiques, traversent l'espace de la chanson qui se réorganise en cette seconde moitié du XIXème siècle, et pour lesquelles l'offre et la demande sont de nature différente. Ce système du marché que l'on vient de schématiser dessine les contours d'un espace de pratiques, d'attentes autour du format général chanson dans lequel le «monde du chansonnier» de la première moitié du siècle, grand pratiquant des timbres de la Clé du Caveau, va devoir chercher ses marques. Il le fera en se démarquant du précédent sous les allures d'une corporation, quasiment d'une «académie», d'une part en en faisant le procès (celui de «fabricants » et de l'entrepreneur qui ose faire carrière, contre l'«artisan» bénévole et dévoué à la chanson), d'autre part en s'octroyant une légitimité de la «tradition française » de l'objet, fondée sur le culte de la coupe et de la rime, l'imitation du savoir-faire des maîtres et le bénévolat. On y dresse la généalogie d'une «vraie » ou de la «bonne chanson» dont les auteurs se proclameront héritiers et dignes successeurs. Le public en est autrement plus modeste : notamment celui d'associations de la mouvance du Caveau ou de la Lice chansonnière regroupées autour d'un savoir-faire chansonnier mis au service de la convivialité, du plaisir de la célébration et de la compétition dans l'entre-soi de cénacles souvent masculins.

Deux mondes, deux esthétiques. L'esthétique dominante au café-concert, de compositeurs familiers des genres de la scène, de la vedette et de la divette, du comique troupier, etc ., expédie au passé révolu cette autre, d'airs à danser et de « petits airs » ronds, carrés ou symétriques qu'affectionnaient les chansonniers du XVIIIème siècle et leurs successeurs, Béranger compris... Esthétique d'un Paulus (Paul Habans, 1845-1908) créant en 1886 la célèbre chanson En revenant de la revue, tandis qu'au Caveau on adapte encore le timbre Eh! Qu'est-ce que ça me fait à Moi ? fort en vogue chez les vieux chansonniers : 


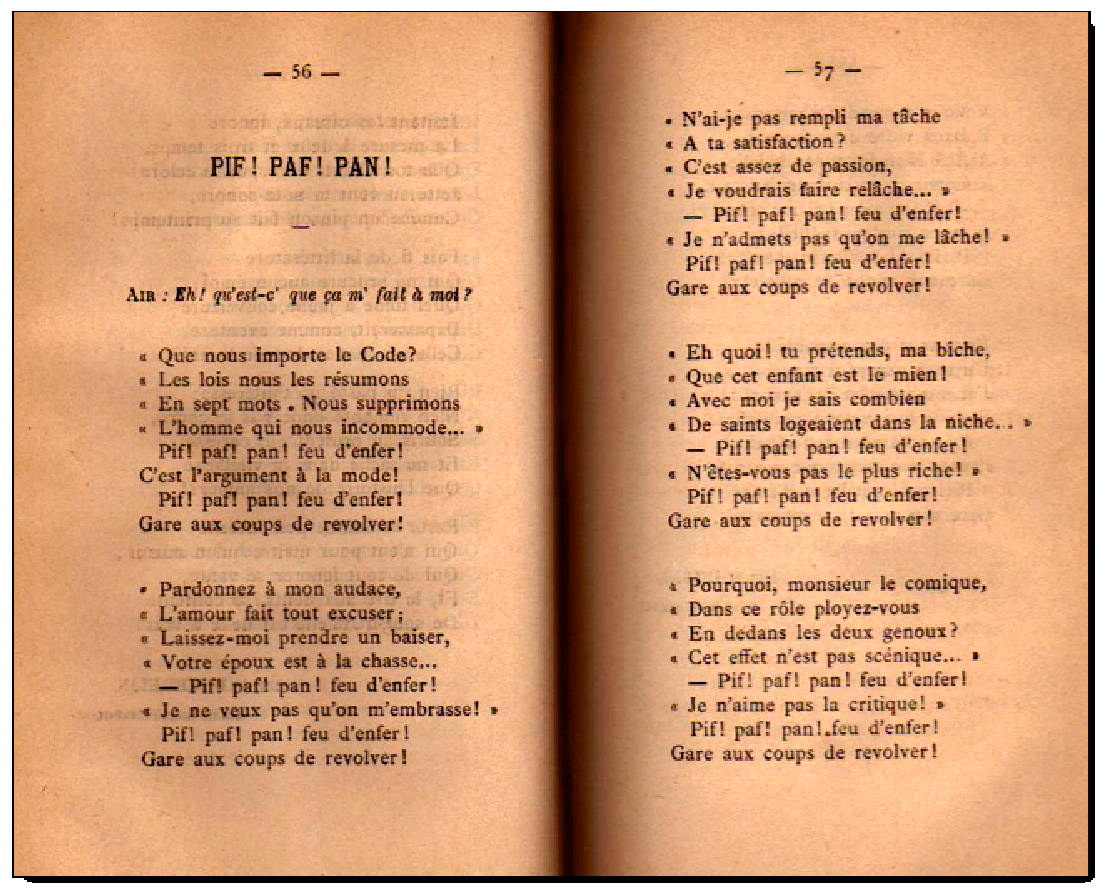

8. Extrait du 52 $2^{\text {ème }}$ volume Le Caveau, 1886. Chanson de l'un de ses membres, Henri Rhéni, pour l'année 1885.

Ce savoir-faire chansonnier fort prisé des associations chansonnières et du bénévolat associatif est également à l'honneur dans les cabarets montmartrois - un système marchand, également -, lieux d'un divertissement plaisamment lettré. Bien des auteurs seront passés par le Caveau ou la Lice. Le chanteur Mayol, se présentant en 1895 pour débuter au Concert parisien avec un répertoire montmartrois, pu s'entendre dire par le directeur d'alors :

Tout cela est trop subtil ; pour se faire tout à fait adopter par le public, il faut lui donner d'abord des choses à sa portée, gaies, de préférence... regarde Dranem / ... Et puis tes chansons montmartroises présentent un grand défaut pour toi : elles sont toutes écrites sur des airs de la «Clé du Caveau » qui n'ont rien de particulièrement folichon et que, de plus, on entend sempiternellement dans tous les cabarets... Pour ces boîtes, c'est une mode spéciale et ça va très bien, mais au café-concert, crois-moi, il faut avant tout les amuser! ... Choisis donc de préférence des musiques alertes et gaies (Les mémoires de Mayol, 1929, p. 89).

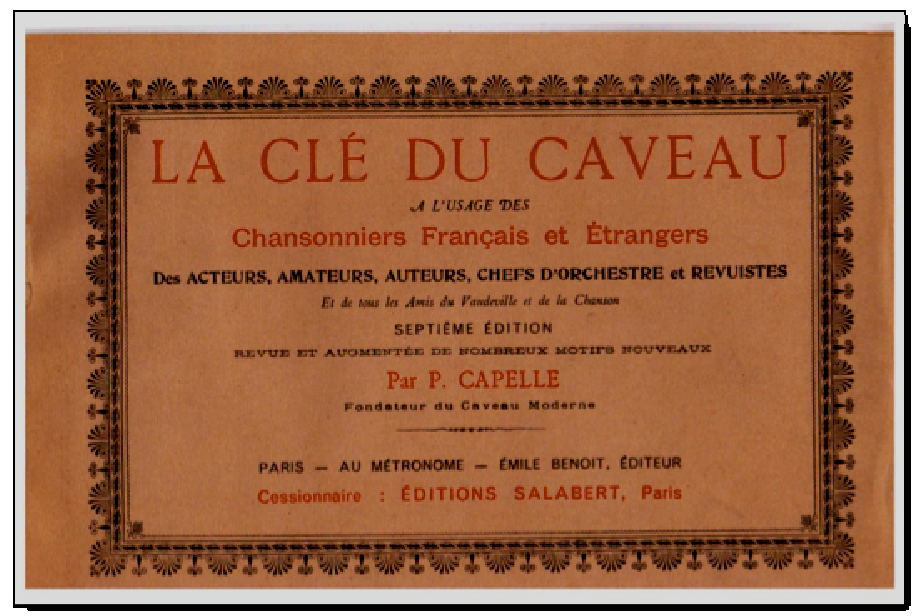

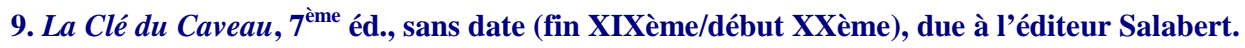


Edition probablement rachetée à l'éditeur Emile Benoît, Sulzbach successeur, qui en avait assuré la ${ }^{\text {ème }}$ éd.

\section{Territoires hymnodiques}

Eventuellement solidaire des précédentes - qu'elles soient associatives ou de cabaret -, une autre «niche» importante d'utilisation de timbres se maintiendra en un secteur que l'on appellera le secteur des pratiques ou même des «territoires hymnodiques». On me permettra de faire état de quelques éléments de ce dossier (il relève d'un travail en cours) susceptibles de rejoindre d'autres interventions de ces journées, en particulier celle de Madame Jacqueline Lalouette sur les « chansons de crises ».

Le pluriel de «territoires» sous-entend ce principe de comparaison entre des formations ou organisations associatives, bref, des collectifs, de type par exemple : églises, partis politiques, syndicats, etc. Les compétences et les exigences de chacun d'eux, c'est-à-dire leurs attendus fondateurs, les finalités qu'ils se donnent (en somme, ce qui définit publiquement leur propre spécificité les uns par rapport aux autres) sont posées ici comme autorisant de les comparer entre eux. En effet, leurs compétences et exigences les placent en compétition sur certains grands principes de société et de démocratie. On se cantonnera à une période d'observation qui ira de la seconde moitié du XIXème siècle (période d'émancipation de grands collectifs politiques et sociaux) aux premières décennies du suivant.

\section{1. «Tous-ensemble ». Une solution profératoire collective}

Tout d'abord, qu'entendre par «hymnodie»? En musicologie, ce terme désigne «l'Art de la composition ou de l'étude des formes musicales des hymnes». Certes. Mais resterait à préciser ce dernier terme comme dans : Hymne à l'Etre suprême, Hymne national, hymne chrétienne, hymne liturgique, hymne au travail, à la liberté, à l'Europe... Prenons cette notion musicologique d'hymnodie comme outil de travail, élargissons-là en partant de la définition que donnent la plupart de nos dictionnaires du terme «hymne»au masculin : «chant en l'honneur de /.../».

L'acte de chanter «tous-ensemble» un format de ce type (chant en l'honneur de /.../», qui resterait à identifier formellement, peut se décrire de manière schématique comme proposant aux membres d'un collectif : (a) un engagement public manifeste (ce qui se donne à voir et à entendre dans l'espace public), (b) une action centrée, (c) une épreuve collective.

(a) Engagement public manifeste. Des répertoires ou formats font l'objet d'une action collective de chant inscrite au répertoires des activités et des marques qu'un groupe considère comme nécessaires à l'entretien de sa cohésion interne et à l'expression de son existence. L'une des particularités thématiques de ces formats résiderait en ce que leurs énoncés portent expressément sur des attributs de grandeur devant caractériser des entités ou valeurs comme, par exemple le Bien public, Dieu, la Nation, la Patrie, la République, la Liberté, le Peuple, etc., entités ou valeurs que fixent ces collectifs à l'horizon de leurs adhérents. Ces formats à pratique collective sont ordonnés pour la manifestation de la considération que tel ou tel collectif entend porter à ces valeurs ou entités, et qu'il place comme principe supérieur commun à l'ensemble de ses membres et comme l'une des conditions d'adhésion au collectif. On appellera ces entités ou ces valeurs posées idéalement à l'horizon des collectifs, entités ou valeurs de Commun idéal. 
L'action de chanter de tels formats constitue par elle-même un acte individuel de manifestation d'adhésion qui a portée d'engagement, d'une part à l'endroit de ce qui est instancié (les entités ou valeurs), d'autre part vis à vis du collectif auquel on participe. Autrement dit, se livrer à l'action collective chantée de tels répertoires peut valoir pour signe d'adhésion ou marque d'appartenance au collectif en question.

(b) Action centrée. Dans certaines manifestations propres au collectif, l'action de chanter collectivement de tels formats peut y être exclusive de toute autre activité (à la différence de chanter en s'occupant à d'autres tâches). Les participants centrent leur attention et leur énergie vocale sur la réalisation pratique de cette action. Ainsi, par exemple, de chanter «tous ensemble » La Marseillaise, debout, statique, parfois le corps figé ; ou de chanter l'Internationale debout, statique, le poing levé.

(c) Epreuve collective. La pratique exercicielle du chant de ce type de format est prioritairement ordonnée à la manifestation du collectif en tant qu'unitaire. Qu'est-ce à dire ? La dimension unitaire dont peut se prévaloir ce collectif n'est pas tant livrée clé en main avec le format à chanter «tous-ensemble » qu'elle «prend», et s'interprète dans le mouvement ou la dynamique même de l'action collective de chanter, lorsque les participants collaborent entre eux, se coordonnent, acceptent des obligations de réciprocité qui organisent le déroulement de l'activité à laquelle ils se livrent. La portée unitaire ou «communautaire » est un idéal à atteindre que se finalise le collectif et qui, comme dans le chant collectif, demande à se réaliser à chaque fois dans la dynamique même de cette action collective de chanter «tous-ensemble ». En ce sens, le Commun idéal porté par le texte du format se réalise au sein du collectif : on «célèbre » la Nation, Dieu, la Liberté, etc., dans la configuration des adhérents ou des sympathisants à ces valeurs ou entités, comme ici à travers l'épreuve de participation de chacun à l'action collective de chanter. L'idéal unitaire ou communautaire s'éprouve dans l'opération collective d'un chanter « tous-ensemble ».

Dans ce contexte, l'action de chant vient constituer une ressource non négligeable, langagière, esthétique et émotionnelle (l'émotion de participer à cette action collective de chanter, et d'entendre soi-même « sonner » ainsi le collectif) : une solution profératoire collective. Le modèle le plus commun de cette solution se présente sous la forme d'une pratique vocale que l'on dira à sociabilité ouverte. Dans ce modèle, il n'y a pas de limites au recrutement des voix, l'un des objectifs résidant dans une mobilisation vocale du plus grand nombre, quels que soient le statut et les compétences musicales de chacun. L'enjeu de ce regroupement vocal est celui, notamment, d'une démonstration, tant au groupe qu'à son extériorité, du caractère unitaire et consensuel du collectif. Une voix mal placée ou une intonation défectueuse n'entrave aucunement l'ambition unitaire ou communautaire de cet acte posé en commun. Le principe de sociabilité ouverte tolère les approximations et la défectuosité (un seuil de tolérance des désajustements vocaux resterait à problématiser au cas par cas). Il existe d'autres modèles de chant collectif ou du moins «à plusieurs », utilisés par ces équipements, mais ils plus axés sur une «civilité chorale » et son modèle fermé (ou semi-ouvert) et à compétence vocale : la chorale ou le chœur.

\section{S'équiper de répertoires}

Replaçons-nous à présent dans le contexte d'un XIXème siècle héritier et continuateur da la période de la Révolution en ce que qu'on y a célébré et théorisé la portée pédagogique et mobilisatrice de la musique, et en premier lieu du chant. Pour maints auteurs de cette période, le chant en général peut constituer précisément en son ordre propre un potentiel de démonstration de force en raison d'une certaine disposition d'enrôlement des 
individus notamment sur un refrain, ce dernier pouvant ainsi devenir support symbolique de ralliement jusqu'à escorter d'autres actions collectives de pression (le refrain de la Carmagnole et de tant d'autres chants collectifs à l'époque).

L'émulation progressive et concurrente dans le cours de ce XIXème siècle de collectifs (partis, syndicats) dont les programmes, les finalités déclarées, entendent concerner des intérêts publics ou communs, l'organisation politique, économique et sociale, morale et religieuse de la démocratie, va continuer la période précédente, ne fût-ce qu'à travers la reprise de répertoires et de timbres pour leur valeur connotative. Chemin faisant, des auteurs, militants chansonniers, y repréciseront la chanson en instrument de militance, en vecteur poético-mélodique de diffusion d'idées, de mobilisation collective des adhérents ou de sympathisants à un Commun idéal touchant au mieux être de la société. Ces collectifs inventeront des types d'organisation, se doteront de répertoires d'actions, d'appareils, d'équipements signalétiques et de scénarisation publique qui permettant la mise en scène du collectif comme dans ses manifestations publiques telles que défilés, meetings. Le chant en sera partie intégrante.

Mais chanter quoi ? Retenons deux grandes dynamiques qui vont œuvrer sur ce «terrain» des répertoires et des territoires hymnodiques.

Et tout d'abord la dynamique générale d'encyclopédisation (par récapitulation, énumération, exemplification) qui s'émancipe, notamment sous des formes patrimoniales - monuments archéologiques, historiques, littéraires, musées, etc. Chemin faisant, elle va s'étendre au domaine du chant et de la chanson; on va établir à cette dernière des inventaires, lui offrir l'honorabilité d'une cause littéraire et l'hospitalité d'une histoire nationale. Quelques repères dans ce travail d' «archéologisation» et de mise en «monuments littéraires» : l'instauration progressive, par exemple d'un corpus de Chants et chansons nationales ou populaires, qui relèvera dans le dernier tiers du siècle de ce que l'on appellera le «folklore ». L'impulsion sera due, pour partie, à la mise sous tutelle scolaire de la musique et principalement du chant, et à l'impérieuse nécessité de se doter de répertoires appropriés. Vers la fin du siècle, Julien Tiersot se fera le chantre de cette rencontre entre chanson folklorique, hymnodie de la Révolution et Ecole laïque : il s'essaiera à une «Lyrique républicaine (10) », à l'instar de ce que l'on appelait alors la « restauration de la musique d'Eglise ». Cette même dynamique d'archéologie et d'anthologies concernant le domaine musical nous vaudra bien d'autres opérations d'inventaires de répertoires, sensiblement à la même époque, comme par exemple les corpus de «chant grégorien (11)» ou d'«hymnes et chansons de la Révolution (12)», de chants patriotiques, militaires. Bref, l'heure serait à une mise en spécialisation de répertoires en anthologies lyriques balisées, à leur titularisation patrimoniale à vocation conservatrice, esthétique, pédagogique, voire cérémonielle.

Quels seront les grands corpus «archéologiques» de formats susceptibles de répondre aux impératifs signalétiques, scénographiques et de conformité aux attendus fondateurs et aux objectifs de ces collectifs à Commun idéal concernant la société ou la nation ? Chansons et hymnes de la période de la Révolution, chansons «nationales » ou populaires, patriotiques, républicaines que l'on réactive lors des événements de 1830, 1848, 1870-71 constitueront un réservoir de formats, de timbres, de formules désignatives disponibles aux réappropriations multiples. Le même processus, à la même époque, sera à l'œuvre par exemple dans le domaine religieux catholique : la Schola Cantorum de Vincent d'Indy, qui soutient l'action des folkloristes ou celles des moines des Solesmes dans la restauration du chant grégorien, proposent un modèle de chant d'église, dont le cantique directement inspiré ou empruntant au modèle grégorien, et qui rompt définitivement avec le passé. 
L'intérêt pour le chant populaire du folklore permettra de sauvegarder quelques niches de timbres anciens comme les noëls.

\section{Pour conclure. La «guerre» des timbres...}

\section{Scénarités vocales}

Dans cette mise en place concurrente de nouveaux répertoires, il faut compter avec le poids symbolique, connotatif et toujours fédérateur de grands modèles comme La Marseillaise, l'Internationale, ou certains autres formats de la période révolutionnaire qui offrent notamment une scénarité vocale appropriée aux grands rassemblements et à la pratique collective du « chant à sociabilité ouverte».

Ainsi : écriture mélodique à large ambitus, ruptures du pattern mélodique par telle ou telle modulation «accidentelle », caractère syllabique, repères assonantiques... Ce type de scénarité vocale sert des réalisations collectives de masse, voire de plein air, en faisant également alterner soliste ou chœur et ensemble des présents dans l'articulation formelle strophes ou couplets et refrain. Il y a là un procédé recherché de dramatisation sonore, éventuellement renforcée par une contribution instrumentale fournie par exemple par des instruments à vent, des percussions. Une cinétique mélodique et rythmique prête son concours, parfois à la manière d'un figuralisme musical : vections ascendantes pour des formules injonctives : "Allons enfants de la Patrie », ou «Marchons! Marchons !» ou encore pour tel shifter, «debout! ». L'attaque du refrain, préparée par le couplet, s'effectue de façon imparable sur des crêtes mélodiques : «Aux armes, Citoyens !», «C'est la lutte finale !», la voix pouvant ainsi donner une intensité maximale, et faire sonner le collectif. Enfin, le caractère tourmenté des profils mélodiques, le poids des injonctifs exhortant à l'action collective et urgente participent de cette dynamique générale de dramatisation sonore et de mobilisation collective. On sait combien La Marseillaise a pu servir de timbre, y compris dans le secteur hymnodique religieux catholique de la première moitié du XIXème siècle (13) (tout comme le Chant du départ, de Nicolas Méhul) : 


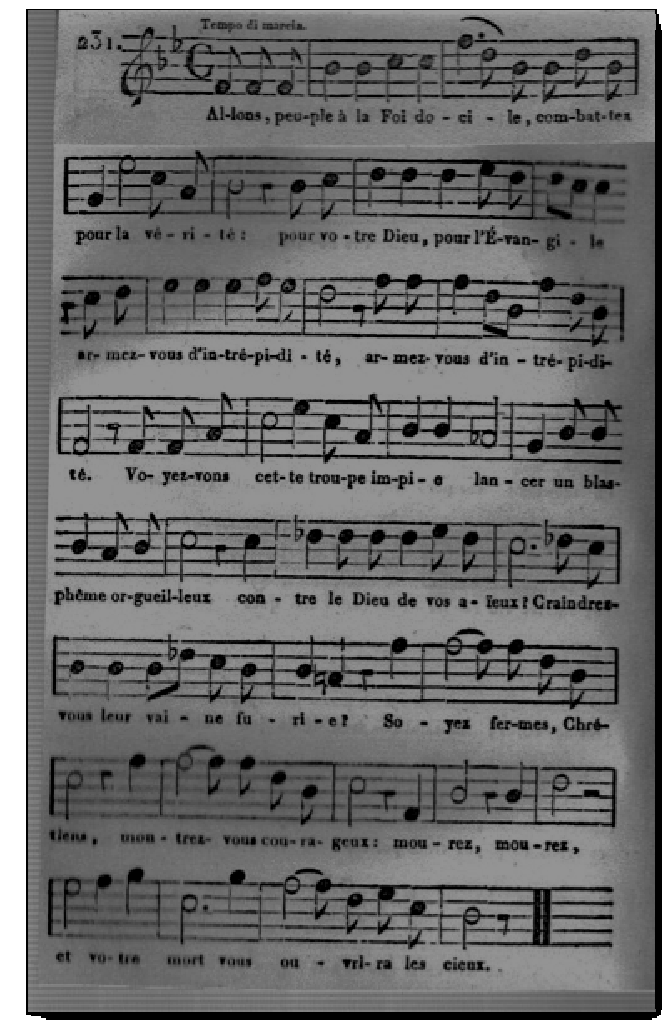

10. Allons, Peuple à la foi docile, cantique sur l'air de La Marseillaise. Recueil de Cantiques spirituels, 1858.

\section{Parodies polémiques}

En résumé, et pour conclure, la formule de «territoires hymnodiques » peut, en particulier dans une perspective de travail sur ce que l'on appelle les «cultures politiques (14)», constituer un outil à la fois musicologique et sociologique ou anthropologique qui permette de problématiser ces équipements musicaux de collectifs. La production hymnodique de tous bords sera foisonnante. Elle ne sera pas seulement le fait d'auteurs reconnus et patentés; elle peut être mise au point entre adhérents ou militants ordinaires (15). Dans cette production, la culture du timbre rappelant de grandes heures de mobilisation ou de grands événements, mais aussi l'emprunt à des succès en vogue jusqu'au café-concert ou au cabaret sera longtemps active, aujourd'hui toujours.

Nous retrouvons ainsi dans cette problématique des territoires hymnodiques, la question même de la parodie, notamment celle, polémique, visant un adversaire par la dérision. Comme procédé de mise au rire publique, les multiples parodies «anti » («anticléricales») ou «contre» («contre-révolutionnaires) qui empruntent, par exemple à un modèle en usage commun chez leurs adversaires - que l'on pense au copieux répertoire anticlérical étudié par J. Lalouette -, ne constituent aucunement une anomalie.

Ces territoires hymnodiques, qu'on les pense comme religieux, politiques, syndicaux, «anarchistes », scolaires, militaires, etc., sont de facto dans un même espace démocratique et sur une même période, en relation de concurrence au sens large, c'est-à-dire aussi bien en relation de neutralité que d'alliance ou polémique. De ce point de vue, la parodie polémique relève du fonctionnement normal de ce «champ » hymnodique. Elle se sert de la position de tel ou tel format dans la pratique d'un collectif adverse ou concurrent pour indigner, pousser à réagir. Dans le même temps, elle sert à réactiver ou conforter une cohésion interne en lui désignant une extériorité par assignation d'un concurrent en adversaire ou en ennemi. 
La pratique de timbre a de beaux jours devant elle. Et sans nul doute pour longtemps...

Jacques Cheyronnaud

Directeur de recherche au CNRS

Shadyc, Ecole des Hautes Etudes en Sciences sociales

Centre de la Vieille Charité - Marseille.

\section{NOTES}

1 Sur ces deux grandes associations historiques organisées autour d'une pratique et d'une culture de la chanson, notamment sur timbre, cf. ici même, notre conférence: Bréviaires de chansonniers.

${ }^{2}$ On emploie à dessein l'expression de volontarisme chansonnier pour ce XIXe siècle (sans doute parlerait-on de nos jours de «chanteur engagé ») en raison du contexte historique dans lequel le format ne représente pas les mêmes enjeux que de nos jours. A certaines périodes comme la Restauration, les restrictions administratives peuvent être sévères : instancier la critique politique, religieuse ou morale, sociale en des formats chanson constitue pour un auteur un risque non négligeable de sanction qui peut aller jusqu'à la prison ; Béranger et bien d'autres chansonniers en feront les frais. Cf. J. Cheyronnaud, "Ce ne sont que des chansons ». Chansons, politique et justice en 1821 », Eidôlon, 82, 2008, pp. 21-30 (numéro spécial : La chanson politique en Europe).

${ }^{3}$ A entendre ici, comme partie (final chanté) d'une pièce, opéra-comique ou autre à l'époque (XVIIIe siècle). Sur ce terme de vaudeville, cf. ici même, notre conférence : Bréviaires de chansonniers.

${ }^{4}$ Cf. Patrice Coirault, Notre chanson folklorique, Paris, Picard, 1942, pp. 210 et 219 (notes de bas de page). Les différents travaux de cet auteur, notamment cet ouvrage, sont incontournables sur la question du timbre. On n'évoquera pas dans la présente intervention la terminologie redoutable des «faux timbres », «faux vrais timbres », «vrais faux timbres », etc.

${ }^{5}$ Cf. ici même notre conférence : Bréviaires de chansonniers.

6 Pierre Laujon (1727-1811), auteur dramatique, vaudevilliste chansonnier. L'auteur consacrera le $4^{\text {ème }}$ tome de ses Euvres choisies (1811) à un état des lieux de la chanson à son époque.

${ }^{7}$ Sur le fonctionnement parodique, parmi les nombreux travaux, cf. C. Abastado, « Situation de la parodie », Cahiers du 20 siècle, 6, 1976, pp 9-37 ; L. Hutcheon, A Theory of Parody. The teachings of Twentieth-Century Art Forms, London, New York, Methuen, 1985 ; D. Sangsue, la relation parodique, Paris, José Corti, 2007.

${ }^{8}$ De nombreux exemples, notamment In : F. P. Bowman, Le Christ romantique, Paris, Droz, 1973 ; du même : Le Christ des barricades, 1789-1848, Paris, Le Cerf, 1987.

${ }^{9}$ Dans l'abondance documentaire sur ce sujet, cf. J.-C. Klein, La chanson à l'affiche. Histoire de la chanson française du café-concert à nos jours, Paris, Du May, 1991. 
${ }^{10}$ Voir sur ce point son ouvrage (J. Tiersot) Les Fêtes et Chants de la Révolution française, Paris, Hachette, 1907 ; cf. J. Cheyronnaud, «Poétique sonore de la République. Le modèle Julien Tiersot », Ethnologie française, 4, 1995, pp. 581-590.

${ }^{11}$ Cf. M. Pérès, J. Cheyronnaud, Les voix du plain-chant, Paris, Desclée de Brouwer, 2001.

${ }^{12} \mathrm{Si}$ le travail de recension documentaire en ce secteur a pu commencer dès le début du XIXème siècle (cf., par ex., Poésies révolutionnaires et contre-révolutionnaires ou Recueil, classé par époques, des Hymnes, Chants guerriers, Chansons républicaines, Odes, satires, Cantiques des missionnaires, etc., etc. Les plus remarquables qui ont paru depuis trente ans, Paris, Librairie historique, 1821, 2 tomes), la fin du siècle et le début du suivant seront marqués par les travaux de Constant Pierre, de J. Tiersot (et quelques controverses entre eux), de Louis Damade, Histoire chantée de la Première République, 1789 à 1799. Chants patriotiques, révolutionnaires et populaires, Paris, P. Schmidt, 1892 et quelques autres.

${ }^{13}$ Sur les innombrables parodies de La Marseillaise, cf. bien sûr : F. Robert, La Marseillaise, Paris, Imprimerie nationale, 1989; également les travaux de J. Lalouette, ainsi que sa conférence ici même, Chansons de crises.

14 Sur ce vaste thème, particulièrement travaillé par l'histoire contemporaine et l'anthropologie politique (à dessein, on n'a pas utilisé ici le terme de «rituel» concernant l'action centrée de chant, pourtant souvent utilisé), cf. en particulier : S. Bernstein (dir.), Les cultures politiques en France, Paris, Seuil, 1999 ; D. Céfaï (dir.), Cultures politiques, Paris, PUF, 2001 ; du même : Pourquoi se mobilise-t-on? Les théories de l'action collective, Paris, La Découverte, 2007.

${ }^{15}$ Sur ce point et plus globalement dans l'abondante documentation, cf. R. Brécy, Florilège de la chanson révolutionnaire, de 1789 au Front populaire, Paris, Editions Hier et demain, 1978 (voir l'ensemble des travaux remarquables de cet auteur); G. Manfredonia, La chanson anarchiste en France des origines à 1914, Paris, L'Harmattan, 1997. On pourra savourer les quelques pages d'Yvonne Quilès, "Georges aime Johnny », In : A. Spire et J. Chancel, La culture des camarades. Que reste-t-il de la culture communiste ?, Paris, Autrement, 1992. Cf. également, ici même notre conférence : Bréviaires de chansonniers. 